\title{
Computer Analysis of Vectorcardiograms in Myocardial Infarction with Special Reference to Polar Vector and Planarity of the QRS and T Loops
}

\author{
Kazunobu Yamauchi, M.D.
}

\section{SUMMARY}

Spatial characteristics of the QRS and T loops in 110 patients with old myocardial infarction were analysed in comparison with 221 normal subjects. Measured were (1) QRS and T polar vectors, (2) initial 20and $30 \mathrm{msec}$ segmental QRS polar vectors, and (3) length, width, thickness, and ratios of width/length and thickness/length of the QRS and T loops in edgewise and broadside projections. Broadside and edgewise projections were obtained by transformation of the reference frame of the Frank lead system to a patient's own frame based on the polar vector.

The recognition rates of abnormality in the QRS and $\mathrm{T}$ polar vectors were $66 \%$ and $60 \%$ of a total of 110 patients with myocardial infarction. The initial $30 \mathrm{msec}$ segmental QRS polar vector showed the highest recognition rate of abnormality in myocardial infarction, i.e., $87 \%$ in anterior myocardial infarction, $100 \%$ in extensive anterior myocardial infarction and $78 \%$ in inferior myocardial infarction. The initial segmental QRS polar vector was abnormally deviated posteriorly and superiorly in inferior myocardial infarction. In anterior myocardial infarction, the initial segmental polar vector was directed inferiorly in more than $50 \%$ of the cases, while the vector in normal subjects was located superiorly and to the left.

The QRS loop of anterior myocardial infarction was significantly smaller in the width and width/length ratio and significantly larger in the thickness and thickness/length ratio than those of the normal. Poor planarity of the QRS loop was one of the characteristics of myocardial infarction, especially of extensive anterior myocardial infarction. The $T$ loop of myocardial infarction was significantly larger in the width/length ratio than that of the normal. More than $50 \%$ of the cases with anterior myocardial infarction showed abnormally wide $T$ loops.

The polar vector was a useful index to characterize the spatial orientation and sense of rotation of the spatial loop. In addition, the initial segmental QRS polar vector represented the mild localized abnormalities of the spatial loop. The loop configuration in space was characterized in edgewise and broadside projections.

From the First Department of Internal Medicine, Nagoya University School of Medicine, 65 Tsurumai-cho, Showa-ku, Nagoya 466, Japan.

Received for publication February 5, 1979. 


\title{
Additional Indexing Words :
}

Frank lead vectorcardiogram Initial $30 \mathrm{msec}$ QRS segmental polar vector Coordinate transformation Broadside and edgewise projections Configuration of the spatial vector loop Recognition rate of abnormalities

\begin{abstract}
GPATIAL characteristics of the vectorcardiographic loops are usually evalu$D$ ated with analysis of the loops projected on the frontal, horizontal, and left sagittal planes. Detailed analyses of the spatial vector loop will be, furthermore, performed by a mathematical procedure, that is, transformation of the reference frame of the Frank lead system to a patient's own frame ${ }^{1,2,}$ based on the polar vector. ${ }^{31}$ Using this procedure, the present study is designed to investigate the characteristics of the spatial loop of myocardial infarction (MI) in comparison with the normal.
\end{abstract}

\section{Materials and Methods}

The "normal" control group was made up of 221 men (age range: 40-69 years) who were screened as to the absence of detectable diseases which might affect the electrocardiogram (ECG) on the bases of history, and routine physical and laboratory examinations. One hundred and ten abnormal cases had a clinically documented myocardial infarction. All patients had records documenting the typical hospital course of acute myocardial infarction, including elevated enzyme studies. The ECG was only used for the classification into 5 subgroups of infarct location: 53 with anterior myocardial infarction (AMI), 16 with extensive anterior myocardial infarction (EAMI), 32 with inferior myocardial infarction (IMI), 4 with inferior-anterior myocardial infarction (IAMI), and 5 with unclassified cases of myocardial infarction. Orthogonal ECG's were recorded on magnetic tape, with the use of the Frank lead system. In the rectilinear coordinate axes of the body, posterior $(\mathrm{Z})$, downwards $(\mathrm{Y})$, and leftward $(\mathrm{X})$ directions were designated as positive. Three $X, Y$, and $Z$ scalar ECG's were digitized at a sampling rate of 500 points/sec. A JEC-6 digital computer with a 4-channel A-D converter, 3 accessory memory units and an XY plotter were used for further data analyses. The determination of the beginning and end points of the QRS and $T$ waves was estimated automatically by a stored computer program.4,5) Beginning and end of QRS and T complexes were taken at the earliest or latest deflection from the base line in any one of the 3 leads.

The normal limits for the following measurements were determined by the formula recommended by Simonson ${ }^{6)}$ using 97.5 and 2.5 percentiles of the values from the 221 normal subjects as the upper and lower limits, respectively.

Measurements:

1) Spatial polar coordinates of the QRS and $T$ polar vectors and the initial 20- and 30-msec QRS segmental polar vectors.")

2) Length, width, thickness, and their mutual ratios of the spatial QRS and T loops. 


\section{Mathematical procedures:}

1) The QRS and T polar vectors and the intial QRS segmental polar vectors.

Let $S_{\mathrm{m}}(\mathrm{m}=1,2, \ldots, \mathrm{N})$ be instantaneous spatial vectors at intervals of 2-msec; then polar vector (PV) for the spatial vector loop is defined approximately by

$$
\begin{aligned}
\mathrm{PV} & =1 / 2 \sum_{\mathrm{m}=1}^{\mathrm{N}-1} \mathrm{SV}_{\mathrm{m}} \times \mathrm{SV}_{\mathrm{m}+1} \\
& =1 / 2 \sum_{\mathrm{m}=1}^{\mathrm{N}-1}\left|\begin{array}{lll}
\mathrm{i} & \mathrm{j} & \mathrm{k} \\
\mathrm{X}_{\mathrm{m}} & \mathrm{Y}_{\mathrm{m}} & \mathrm{Z}_{\mathrm{m}} \\
\mathrm{X}_{\mathrm{m}+1} & \mathrm{Y}_{\mathrm{m}+1} & \mathrm{Z}_{\mathrm{m}+1}
\end{array}\right|
\end{aligned}
$$

where $i, j$, and $k$ are unit vectors along the positive $X, Y$, and $Z$ axes, respectively; $\mathrm{X}_{\mathrm{m}}, \mathrm{Y}_{\mathrm{m}}$, and $\mathrm{Z}_{\mathrm{m}}$ are 3 orthogonal components of the $\mathrm{m}$-th spatial instantaneous vector. The intial 20 - and $30-\mathrm{msec}$ QRS segmental polar vectors are defined when $\mathrm{N}=10$ and 15 , respectively. The $\mathrm{T}$ polar vectors are calculated from the beginning of $\mathrm{S}-\mathrm{T}$ segment to the end of the $\mathrm{T}$ waves. Spatial polar coordinates, i.e., azimuth, elevation, and magnitude are calculated by the following formulae:

$$
\begin{aligned}
& \text { Azimuth } \quad\left(\mathrm{H}^{\circ}\right)=\tan ^{-1} \quad \mathrm{Vz} / \mathrm{Vx}, \\
& \text { Elevation } \quad\left(\mathrm{V}^{\circ}\right)=\cos ^{-1} \quad \mathrm{Vy} / \sqrt{\mathrm{Vx}^{2}+\mathrm{V}^{2}+\mathrm{Vz}^{2}}, \\
& \text { Magnitude }(\mathrm{Mag})=\sqrt{\mathrm{Vx}^{2}+\mathrm{V}^{2}+\mathrm{Vz}^{2}}
\end{aligned}
$$

where $\mathrm{Vx}, \mathrm{Vy}$, and $\mathrm{Vz}$ are 3 orthogonal components of polar vectors, i.e., the total area of the loop in the left sagittal, horizontal, and frontal projections, respectively. For the azimuth $\left(\mathrm{H}^{\circ}\right)$ angle, zero degree is situated to the left, plus 90 degree is directed anteriorly and minus 90 degree posteriorly. In the elevation $\left(\mathrm{V}^{\circ}\right)$ angle, the inferior and superior directions are taken as zero and 180 degrees, respectively.

2) Length, width, and thickness of the spatial loop (Fig. 1).

The reference frame of the Frank lead system was transformed to a patient's own frame to calculate length (a), width (b), and thickness (c) of the spatial loop. In the new reference frame, axis $\mathrm{X}^{\prime}$ is the direction of the maximal spatial vector. Axis $\mathrm{Y}^{\prime}$ is the direction of the polar vector which is perpendicular to an approximated plane for the spatial loop. ${ }^{7), 8}$ The approximated plane (VxX+VyY+ $\mathrm{VzZ}=0$ ) passes the origin. Axis $\mathrm{Z}^{\prime}$ is designated to be at a right angle to axes $\mathrm{X}^{\prime}$ and $Y^{\prime}$. Let the position of a point on a spatial loop referred to the lead frame be $\left(\mathrm{X}_{\mathrm{m}}, \mathrm{Y}_{\mathrm{m}}, \mathrm{Z}_{\mathrm{m}}\right)$ and direction cosines of the spatial maximal vector, the polar vector and the vector at a right angle to the former 2 be $\left(\lambda_{1}, \mu_{1}, \nu_{1}\right),\left(\lambda_{2}, \mu_{2}, \nu_{2}\right)$, and $\left(\lambda_{3}\right.$, $\left.\mu_{3}, \nu_{3}\right)$, respectively, the position of the point $\left(\mathrm{X}_{\mathrm{m}}{ }^{\prime}, \mathrm{Y}_{\mathrm{m}}{ }^{\prime}, \mathrm{Z}_{\mathrm{m}}{ }^{\prime}\right)$ on the loop referred to the new frame is represented by

$$
\begin{aligned}
& \mathrm{X}_{\mathrm{m}}{ }^{\prime}=\lambda_{1} \mathrm{X}_{\mathrm{m}}+\mu_{1} \mathrm{Y}_{\mathrm{m}}+\nu_{1} \mathrm{Z}_{\mathrm{m}}, \\
& \mathrm{Y}_{\mathrm{m}}{ }^{\prime}=\lambda_{2} \mathrm{X}_{\mathrm{m}}+\mu_{2} \mathrm{Y}_{\mathrm{m}}+\nu_{2} \mathrm{Z}_{\mathrm{m}}, \\
& \mathrm{Z}_{\mathrm{m}}{ }^{\prime}=\lambda_{3} \mathrm{X}_{\mathrm{m}}+\mu_{3} \mathrm{Y}_{\mathrm{m}}+\nu_{3} \mathrm{Z}_{\mathrm{m}} .
\end{aligned}
$$

The broadside view of the spatial loop is expressed by time series of $\mathrm{X}_{\mathrm{m}}{ }^{\prime}$ and $\mathrm{Z}_{\mathrm{m}}{ }^{\prime}$ and edgewise view by $\mathrm{X}_{\mathrm{m}}{ }^{\prime}$ and $\mathrm{Y}_{\mathrm{m}}{ }^{\prime}$, or $\mathrm{Y}_{\mathrm{m}}{ }^{\prime}$ and $\mathrm{Z}_{\mathrm{m}}{ }^{\prime}$, respectively. Length, width and thickness are defined as distances between the 2 most distant parts of the spatial 


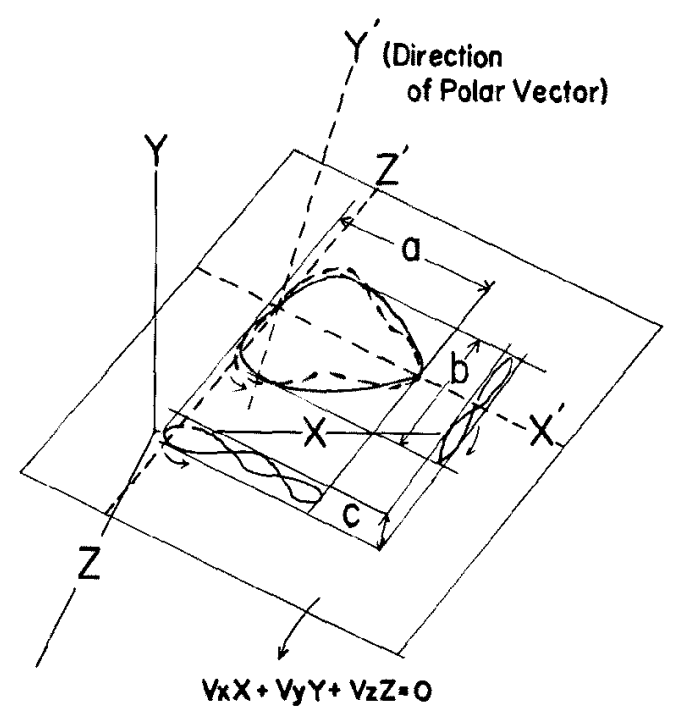

Fig. 1. Methods of obtaining "broadside" and "edgewise" QRS loops. A spatial dotted QRS loop in X Y Z axes of the Frank lead system was transformed in $X^{\prime} Y^{\prime} Z^{\prime}$ axes of a patient's own frame. The broadside loop in the middle is a projection of the spatial loop on the approximated QRS plane $(\mathrm{VxX}+\mathrm{Vy} \mathrm{Y}+\mathrm{VzZ}=0)$. The other 2 are edgewise QRS loop projections. $\mathrm{Y}^{\prime}$ is the direction of the polar vector. $\mathrm{X}^{\prime}$ is the direction of the maximal spatial vector on the approximated plane. Length (a), width (b), and thickness (c) are distances between the 2 most distant parts of the spatial loops along with $\mathrm{X}^{\prime}, \mathrm{Z}^{\prime}$, and $\mathrm{Y}^{\prime}$ axes, respectively. For the method of coordinate transformation, see text.

loops along with $\mathrm{X}^{\prime}, \mathrm{Z}^{\prime}$, and $\mathrm{Y}^{\prime}$ axes, respectively. The ratio of thickness/length was taken as an indicator of the spatial loop planarity. ${ }^{1,93}$

\section{Results}

Normal 95 percentile ranges for the direction (azimuth and elevation) of the QRS, initial QRS segmental and $\mathrm{T}$ polar vectors were obtained. The measurements were considered abnormal when they were found outside of the normal ranges (Table I, Fig. 2). The group of 69 cases of old anterior wall myocardial infarction (AMI +EAMI) showed a wide scatter of directions of the QRS polar vector. Forty-five out of 69 cases $(65 \%)$ were abnormal in the direction of the vector. Twenty-three of 32 patients with inferior myocardial infarction (72\%) showed abnormal QRS polar vectors which oriented more posteriorly than the normal $\left(\mathrm{H}^{\circ}:-52.1^{\circ} \pm 18.3^{\circ}\right.$, IMI, vs $-8.2^{\circ} \pm 27.9^{\circ}$, normal, $\mathrm{V}^{\circ}: 142.1^{\circ} \pm 14.8^{\circ}$, IMI, vs $137.9^{\circ} \pm 12.3^{\circ}$, normal). The cases with inferior myocardial infarction showed a tendency to cluster in the polar vector direction (Fig. 2). 
Table I. Normal Ranges of the Directions of the Polar Vectors and Percentages of the Cases Outside the Normal Ranges in Myocardial Infarction (MI) (degree)

\begin{tabular}{|c|c|c|c|c|}
\hline & $\begin{array}{l}\text { QRS polar } \\
\text { vector }\end{array}$ & $\begin{array}{l}\text { Initial } 20 \mathrm{msec} \\
\mathrm{QRS} \text { segmental } \\
\text { polar vector }\end{array}$ & $\begin{array}{l}\text { Initial } 30 \text { msec } \\
\text { QRS segmental } \\
\text { polar vector }\end{array}$ & $T$ polar vector \\
\hline Normal $\mathrm{H}^{\circ}$ & $(-52 \sim 0 \sim 60)$ & $(-160 \sim 0 \sim 160)$ & $(-56 \sim 0 \sim 70)$ & $(-62 \sim 0 \sim 22)$ \\
\hline $\mathrm{N}=221 \quad \mathrm{~V}^{\circ}$ & $(112 \sim 164)$ & $(50 \sim 172)$ & $(114 \sim 166)$ & $(95 \sim 153)$ \\
\hline AMI & & & & \\
\hline $\mathrm{N}=53$ & $62 \%$ & $38 \%$ & $87 \%$ & $45 \%$ \\
\hline EAMI & & & & \\
\hline$N=16$ & $75 \%$ & $44 \%$ & $100 \%$ & $81 \%$ \\
\hline IMI & & & & \\
\hline$N=32$ & $72 \%$ & $44 \%$ & $78 \%$ & $72 \%$ \\
\hline IAMI & & & & \\
\hline$N=4$ & $100 \%$ & $25 \%$ & $100 \%$ & $75 \%$ \\
\hline $\mathrm{U}$ & & & & \\
\hline$N=5$ & $20 \%$ & $20 \%$ & 0 & $60 \%$ \\
\hline Total & & & & \\
\hline $\mathrm{N}=110$ & $66 \%$ & $39 \%$ & $83 \%$ & $60 \%$ \\
\hline
\end{tabular}

The normal 95 percentile ranges are shown in parentheses. $\mathrm{H}^{\circ}=$ azimuth; $\mathrm{V}^{\circ}=$ elevation; $\mathrm{AMI}=$ anterior $\mathrm{MI}$; $\mathrm{EAMI}=$ extensive anterior $\mathrm{MI}$; IMI =inferior $\mathrm{MI}$; IAMI=inferior anterior $\mathrm{MI}$; $\mathrm{U}=$ unknown location.

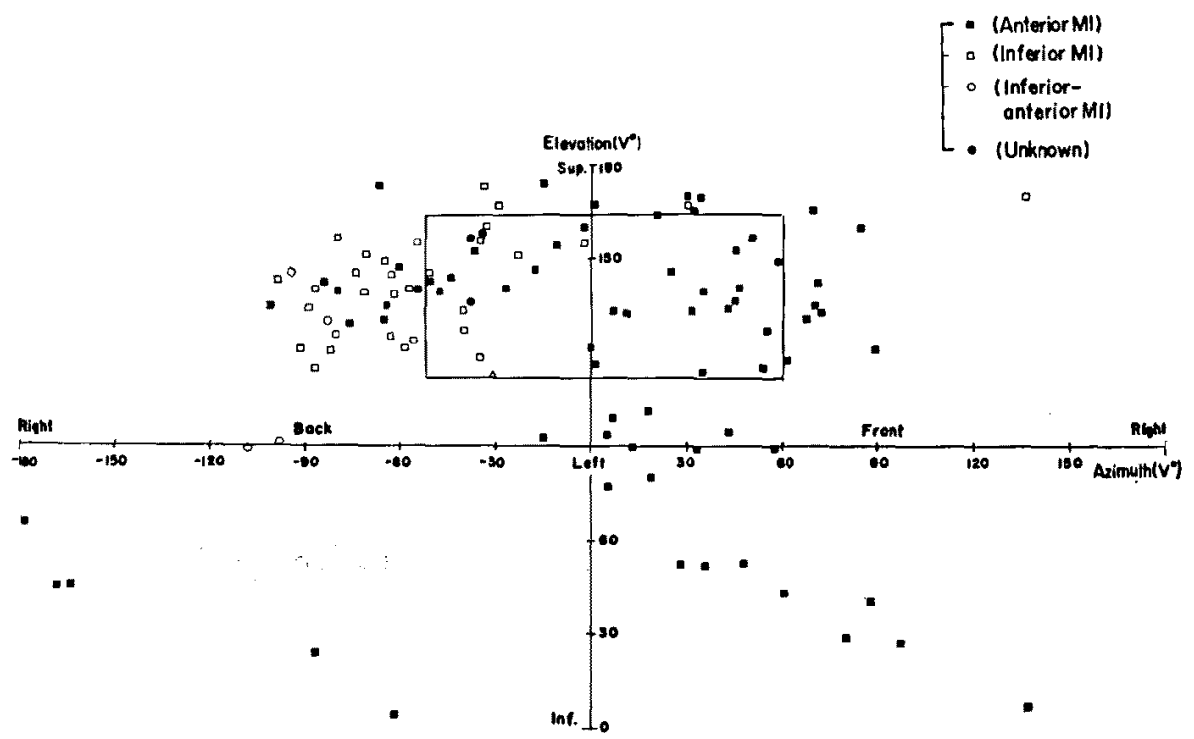

Fig. 2. Orientation of the mean $Q R S$ polar vector in myocardial infarction. The rectangle area is the normal range for azimuthal and elevational angles of the vector. The wide spread of the QRS polar vector is shown in anterior myocardial infarction. In inferior myocardial infarction, the main tendency in deviation from the normal is in posterior direction. 
When the initial 30-msec QRS segmental polar vector was used (Table I, Fig. 3), the cases included in the normal range (false negatives) were appreciably decreased. The recognition rate of abnormality of the initial 30 msec segmental polar vector was $87 \%$ in AMI, $78 \%$ in IMI, and $100 \%$ in EAMI and IAMI. This initial segmental polar vector was directed inferiorly in $57 \%$ of the cases of anterior myocardial infarction (AMI + EAMI), although a specific cluster in the direction was not found. In inferior myocardial infarction, the mean direction of the initial segmental polar vector was in the superior and posterior direction $\left(\mathrm{H}^{\circ}:-71.9^{\circ} \pm 18.3^{\circ}, \mathrm{IMI}\right.$, vs $-5.7^{\circ} \pm$ $30.2^{\circ}$, normal, $\mathrm{V}^{\circ}$ : $131.3^{\circ} \pm 18.5^{\circ}$, IMI, vs $142.1^{\circ} \pm 13.9^{\circ}$, normal). Of the total of 110 patients, 91 cases $(83 \%)$ fell outside the normal range in the initial 30-msec QRS segmental polar vectors. The lowest recognition rate of abnormality was found in the initial 20 -msec QRS segmental polar vectors, probably due to the wide normal range of the vector. The $\mathrm{T}$ polar vector was found abnormal in a large percentage of EAMI (81\%), IMI (72\%), and IAMI $(75 \%)$.

Anterior myocardial infarction (AMI +EAMI) showed a smaller mean magnitude of the QRS polar vector than the normal $\left(1.07 \pm 0.73 \mathrm{mV}^{2}, \mathrm{AMI}+\right.$ EAMI, vs $1.29 \pm 0.64 \mathrm{mV}^{2}$, normal, $\left.\mathrm{p}<0.05\right)$. The initial 30 -msec segmental

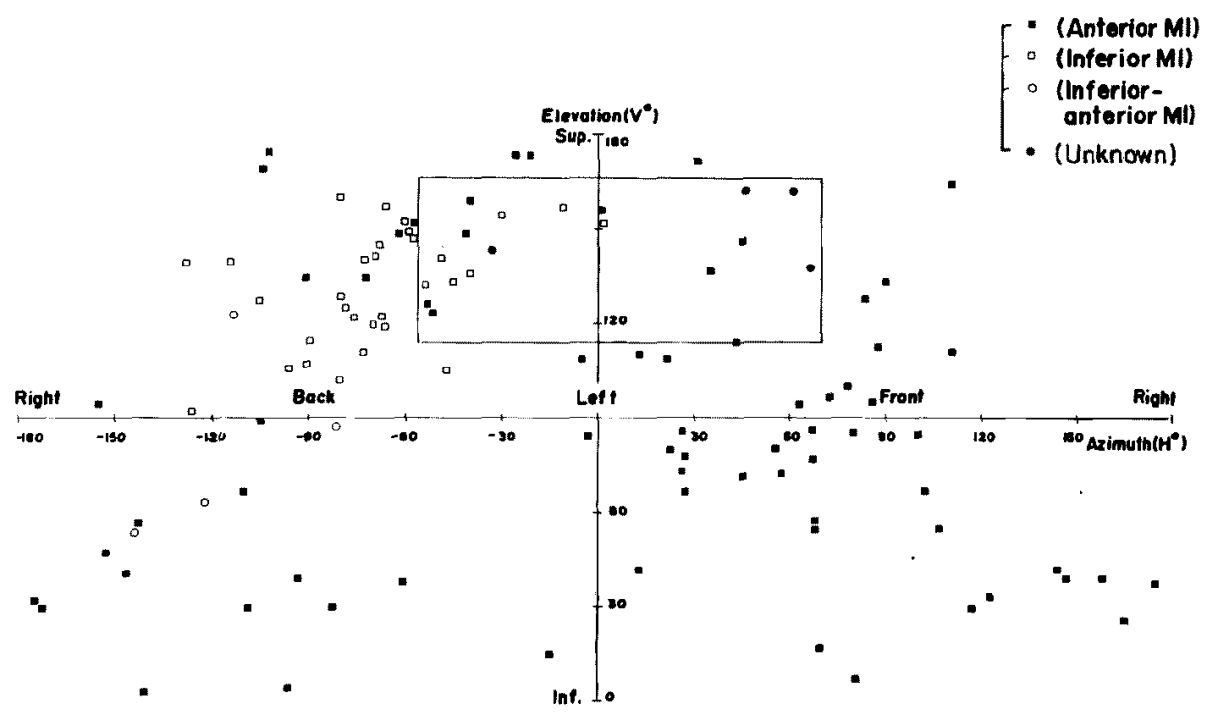

Fig. 3. Orientation of the initial 30 -msec QRS segmental polar vector in myocardial infarction. The normal range is the rectangle area depicted with a solid line. Note the significant decrease of the false negatives compared with the mean QRS polar vector. In anterior myocardial infarction, the initial 30-msec segmental QRS polar vector was directed inferiorly in 57\% of the cases. 
Table II, The Means (M), Standard Deviations (SD), Normal Ranges and Percentages of the Cases Outside the Normal Ranges in the

Parameters of QRS Loops Measured in Edgewise and Broadside Projections

\begin{tabular}{|c|c|c|c|c|c|c|c|c|c|c|}
\hline & \multicolumn{2}{|c|}{$\mathrm{a}(\mathrm{mV})$} & \multicolumn{2}{|c|}{$\mathrm{b}(\mathrm{mV})$} & \multicolumn{2}{|c|}{$c(\mathrm{mV})$} & \multicolumn{2}{|c|}{$b / a$} & \multicolumn{2}{|c|}{$c / a$} \\
\hline & $\mathbf{M}$ & $\mathrm{SD}$ & $\mathbf{M}$ & $\mathrm{SD}$ & $\mathbf{M}$ & $\mathrm{SD}$ & $\mathbf{M}$ & $\mathrm{SD}$ & $\mathrm{M}$ & $\mathrm{SD}$ \\
\hline Normal & 1.85 & 0.43 & 1.17 & 0.41 & 0.25 & 0.10 & 0.66 & 0.25 & 0.14 & 0.06 \\
\hline $\mathrm{N}=221$ & $(1.15 \sim$ & $2.80)$ & $(0.52 \sim$ & $2.11)$ & $(0.11 \sim$ & $0.47)$ & $(0.24$ & $1.18)$ & $(0.05 \sim$ & $0.30)$ \\
\hline $\mathrm{AMI}$ & 1.95 & 0.58 & $0.90^{* *}$ & 0.39 & $0.39 * *$ & 0.23 & $0.49 * *$ & 0.24 & $0.22 * *$ & 0.15 \\
\hline$N=53$ & \multicolumn{2}{|c|}{$15 \%$} & \multicolumn{2}{|c|}{$17 \%$} & \multicolumn{2}{|c|}{$17 \%$} & \multicolumn{2}{|c|}{$8 \%$} & \multicolumn{2}{|c|}{$9 \%$} \\
\hline EAMI & 2.07 & 0.57 & $0.86 * *$ & 0.40 & $0.41 * *$ & 0.18 & $0.44^{* *}$ & 0.18 & $0.20 * *$ & 0.07 \\
\hline $\mathrm{N}=16$ & \multicolumn{2}{|c|}{$13 \%$} & \multicolumn{2}{|c|}{$6 \%$} & \multicolumn{2}{|c|}{$31 \%$} & \multicolumn{2}{|c|}{$6 \%$} & \multicolumn{2}{|c|}{$6 \%$} \\
\hline IMI & 2.10 & 0.65 & 1.27 & 0.53 & $0.32^{*}$ & 0.16 & 0.63 & 0.25 & 0.16 & 0.07 \\
\hline$N=32$ & \multicolumn{2}{|c|}{$16 \%$} & \multicolumn{2}{|c|}{$9 \%$} & \multicolumn{2}{|c|}{$22 \%$} & \multicolumn{2}{|c|}{$3 \%$} & \multicolumn{2}{|c|}{$3 \%$} \\
\hline
\end{tabular}

The normal 95 percentile ranges arc shown in parentheses. AMI=anterior $\mathrm{MI}$; EAMI = extensive anterior $\mathrm{MI}$; $\mathrm{IMI}=$ inferior $\mathrm{MI} ; \mathrm{a}=$ length $; \mathrm{b}=$ width ; $\mathrm{c}=$ thickness.

${ }^{*} \mathrm{p}<0.05,{ }^{* *} \mathrm{p}<0.01,{ }^{* * *} \mathrm{p}<0.001$.

polar vector in anterior myocardial infarction was also significantly smaller than that in the normal $\left(0.10 \pm 0.12 \mathrm{mV}^{2}\right.$, AMI +EAMI, vs $0.22 \pm 0.14 \mathrm{mV}^{2}$, normal, $\mathrm{p}<0.001)$. However, there was no significant magnitude differences in the QRS $\left(1.52 \pm 0.88 \mathrm{mV}^{2}\right.$, IMI, vs $1.29 \pm 0.64 \mathrm{mV}^{2}$, normal, NS) and initial 30 -msec segmental $\left(0.20 \pm 0.16 \mathrm{mV}^{2}\right.$, IMI, vs $0.22 \pm 0.14 \mathrm{mV}^{2}$, normal, NS) polar vectors between inferior myocardial infarction and the normal.

The means, standard deviations and normal limits of the parameters in broadside and edgewise projections are given in Tables II and III. In the QRS loop (Table II), anterior myocardial infarction (AMI +EAMI) showed significantly smaller width and width/length ratios $(\mathrm{p}<0.01)$ and significantly larger thickness and thickness/length ratios $(\mathrm{p}<0.01)$ than the normal. Inferior myocardial infarction showed a tendency to have larger mean values of thickness/length ratios. Thickness was found abnormal in a large percentage of extensive anterior myocardial infarction $(31 \%)$. Poor planarity of the QRS loop is one of the characteristics of myocardial infarction, especially of extensive anterior myocardial infarction.

Representative broadside and edgewise views of the QRS and T loops in normal, extensive anterior myocardial infarction and inferior myocardial infarction are given in Fig. 4. The normal QRS loop showed excellent planarity. The mid portions of efferent and afferent limbs of the $Q R S$ loop bulged in the opposite direction to the polar vector in extensive anterior myocardial infarction, on the contrary, in the polar vector direction in inferior myocardial infarction. This tendency in the edgewise QRS loop was noted 

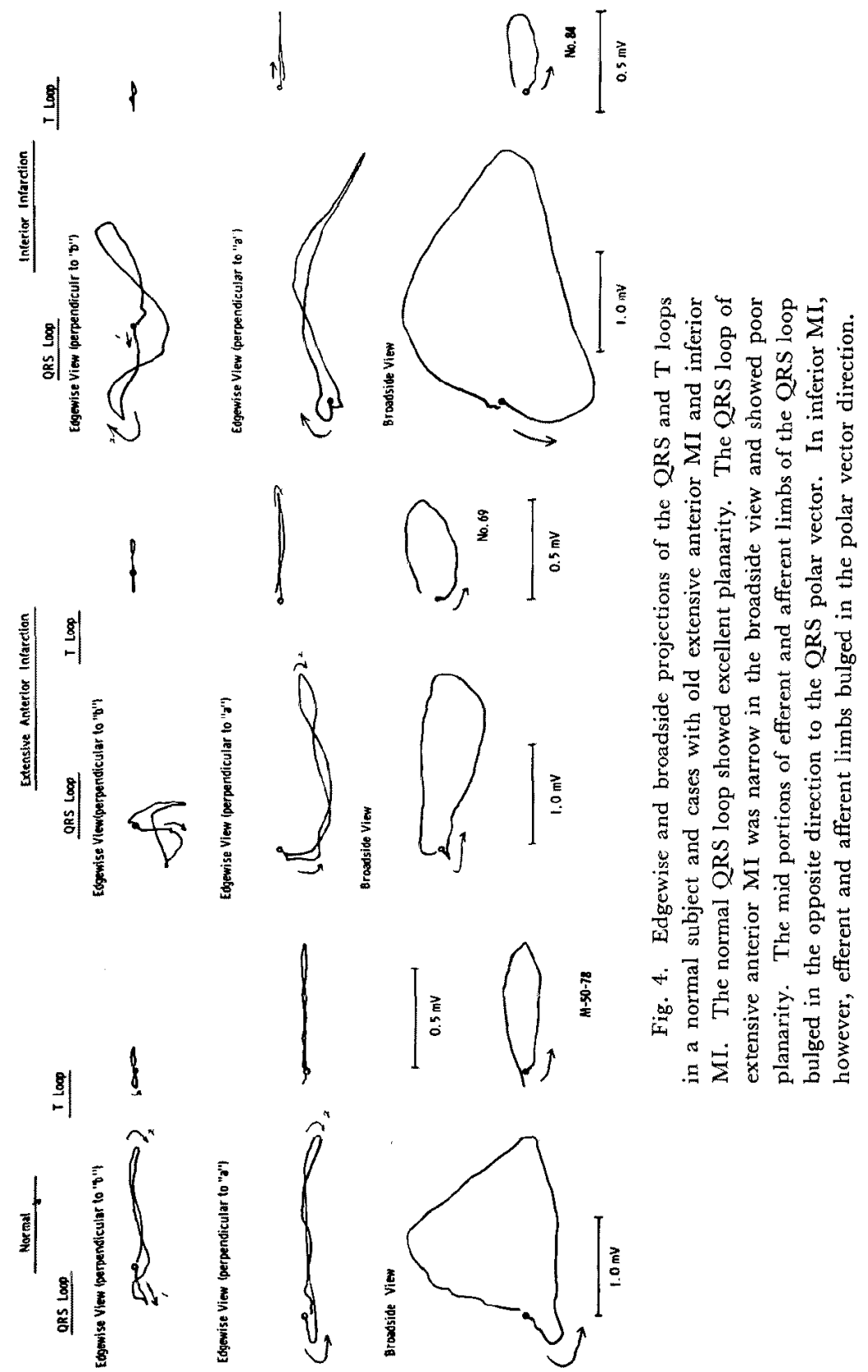

in $44 \%$ of anterior myocardial infarction (AMI $+\mathrm{EAMI}$ ) and $48 \%$ of inferior myocardial infarction.

In the $\mathrm{T}$ loop (Table III), patients with myocardial infarction showed smaller mean values in length and larger values in width. The width/length 
Table III. The Means (M), Standard Deviations (SD), Normal Ranges and Percentages of the Cases Outside the Normal Ranges in the Parameters of T Loops Measured in Edgewise and Broadside Projections

\begin{tabular}{|c|c|c|c|c|c|c|c|c|c|}
\hline & $\mathbf{a}(\mathrm{mV})$ & \multicolumn{2}{|c|}{$\mathrm{b}(\mathrm{mV})$} & \multicolumn{2}{|c|}{ c. $(\mathrm{mV})$} & \multicolumn{2}{|c|}{$\mathrm{b} / \mathrm{a}$} & \multicolumn{2}{|c|}{$\mathrm{c} / \mathrm{a}$} \\
\hline & SD & $\mathbf{M}$ & $\mathrm{SD}$ & $\mathbf{M}$ & $\mathrm{SD}$ & $\mathbf{M}$ & SD & $\mathbf{M}$ & SD \\
\hline Normal & $0.57 \quad 0.18$ & 0.14 & 0.05 & 0.04 & 0.02 & 0.27 & 0.11 & 0.08 & 0.06 \\
\hline $\mathrm{N}=221$ & $(0.25 \sim 0.94)$ & $(0.05 \sim$ & 0.26 ) & $(0.01 \sim$ & 0.09 ) & $(0.10 \sim$ & $0.53)$ & $(0.03 \sim$ & $0.24)$ \\
\hline AMI & $0.41 * * \quad 0.18$ & $0.20 * *$ & 0.10 & 0.04 & 0.02 & $0.56^{* * *}$ & 0.31 & $0.12^{* * *}$ & 0.09 \\
\hline$N=53$ & $25 \%$ & \multicolumn{2}{|c|}{$28 \%$} & \multicolumn{2}{|l|}{0} & \multicolumn{2}{|c|}{$51 \%$} & \multicolumn{2}{|c|}{$11 \%$} \\
\hline EAMI & $0.41 * * \quad 0.11$ & $0.23 * *$ & 0.09 & 0.05 & 0.02 & $0.56^{* * *}$ & 0.18 & $0.14^{* * *}$ & 0.10 \\
\hline$N=16$ & $6 \%$ & \multicolumn{2}{|c|}{$44 \%$} & \multicolumn{2}{|c|}{$6 \%$} & $560^{\circ}$ & & \multicolumn{2}{|c|}{$13 \%$} \\
\hline IMI & $0.45 * * \quad 0.21$ & 0.14 & 0.07 & 0.04 & 0.02 & $0.37 * * *$ & 0.13 & 0.10 & 0.06 \\
\hline $\mathrm{N}=32$ & $19 \%$ & \multicolumn{2}{|c|}{$9 \%$} & \multicolumn{2}{|c|}{$6 \%$} & \multicolumn{2}{|c|}{$13 \%$} & \multicolumn{2}{|c|}{$6 \%$} \\
\hline
\end{tabular}

The normal 95 percentile ranges are shown in parentheses. AMI=anterior MI; EAMI= extensive anterior $\mathrm{MI} ; \mathrm{IMI}=$ inferior $\mathrm{MI} ; \mathrm{a}=$ length $; \mathrm{b}=$ width ; $\mathrm{c}=$ thickness.

${ }^{* *} \mathrm{p}<0.01,{ }^{* * *} \mathrm{p}<0.001$.

ratios were significantly larger in myocardial infarction than the normal $(p<0.001)$. More than $50 \%$ of the cases of anterior myocardial infarction (AMI + EAMI) showed abnormally wide $\mathrm{T}$ loops. There were no significant differences in the $\mathrm{T}$ loop thickness between normal and myocardial infarction. However, thickness/length ratios were significantly larger in anterior myocardial infarction than in the normal due to the decreased length.

\section{Discussion}

Schellong first demonstrated in 1936 that the spatial QRS loop of normal subjects nearly formed a plane termed "the major QRS plane"." The spatial orientation of the plane can be defined by a vector perpendicular to the plane, which was called, by Burger and Vaane, the polar vector. ${ }^{3)}$ The magnitude of this vector corresponds to the area of the loop in the major QRS plane i.e., "broadside" projection. The polar vector direction is such that it points away from the projection when the loop rotation is counter-clockwise. $^{3), 9)}$ This vector, which is a spatial counterpart of Einthoven's manifest QRS axis in the frontal plane, ${ }^{101}$ gives a criterion characterizing the position of the QRS plane in space as well as the sense of inscription of the loop, ${ }^{1 /}$ and has been shown to be useful for screening normal and abnormal records. ${ }^{1)}$

Pipberger et al,11,12) however, pointed that the polar vector direction was not measurably better than the instantaneous vector in the differential diagnosis. In the conventional electrocardiographic and vectorcardiographic 
diagnosis of myocardial infarction, the diagnosis has been based on the abnormalities of initial QRS complex. This very initial change is, however, not clearly defined by means of the so-called mean polar vector which indicates the direction of the whole spatial loop and does not include the time information. ${ }^{11)}$ Therefore, the QRS loop was segmentalized and the initial segmental polar vector was analysed. The significance of this initial "segmental polar vector" was reported by Okamoto and Simonson in various abnormal cardiac diseases. ${ }^{7}$ Furthermore, Yasui and co-workers ${ }^{13}$ obtained the areal velocity curve which was the serial plot of the triangular area (i.e., 3 components of the segmental polar vector) of the QRS loop, revealing the distortion of the initial portion of the QRS loop as well as the increase of high frequency components of the QRS loop in myocardial infarction.

In the present study, emphasis was placed on the statistical recognition rate of abnormality using newly established normal ranges of the initial segmental and mean QRS and $\mathrm{T}$ polar vectors for Japanese males.

In anterior myocardial infarction, $65 \%$ of the cases displayed abnormal directions of the QRS polar vector. As reported by Pipberger, ${ }^{1)}$ a wide scatter of direction was not appreciably decreased when the cases were separated according to the locations of infarction. The cases included in the normal ranges (false negatives) of the QRS polar vector were 24 out of 69 patients with anterior myocardial infarction $(35 \%)$. This percentage is rather high as compared with that of inferior myocardial infarction $(25 \%)$. The cases remaining in the normal ranges were considered to have significant changes in the initial portion of the QRS loop alone, and these changes did not lead to a significant shift of the QRS polar vector (i.e., QRS plane). When the initial 30-msec QRS segmental polar vector was used, the recognition rate of abnormality was improved to $89 \%$ in anterior myocarial infraction. This improvement is probably due to the inferiorly-directed initial segmental QRS polar vector (Fig. 3) which corresponds with the "indentation" in the initial portion of the QRS loop.

In contrast to inferior myocardial infarction, anterior myocardial infarction showed significant magnitude differences in both the initial $30-\mathrm{msec}$ segmental and mean QRS polar vectors. The mean values and standard deviations $\left(\mathrm{mV}^{2}\right.$ ) of the 3 components of the initial 30-msec segmental QRS polar vector were $V_{x}=0.005 \pm 0.069, V_{y}=-0.009 \pm 0.100, V_{z}=0.022 \pm 0.065$ in $\mathrm{AMI}+\mathrm{EAMI}, \mathrm{vs} \mathrm{Vx}=0.121 \pm 0.102, \mathrm{Vy}=0.171 \pm 0.113, \mathrm{Vz}_{z}=-0.015 \pm 0.055$ in normal. Those of the mean QRS polar vector were $V x=0.345 \pm 0.535$, $V_{y}=0.302 \pm 0.854, \quad V z=0.170 \pm 0.567$, in $A M I+E A M I, \quad$ vs $V x=0.779 \pm$ $0.485, \mathrm{Vy}=0.955 \pm 0.494, \mathrm{Vz}=-0.183 \pm 0.388$ in normal. This result indicates that the smaller magnitude of the polar vectors in anterior myocardial 
infarction was mainly due to the decrease of the loop area in the sagittal and horizontal planes in which the QRS loop of anterior myocardial infarction was usually characterized.

Posterior deviation of the polar vector which corresponds with the clockwise inscription of the frontal QRS loop is the characteristic of inferior myocardial infarction. Here, a problem is posed by differentiation against typical right ventricular hypertrophy which also shows clockwise rotation of the QRS loop in the frontal plane. ${ }^{14}$ However, the polar vector in right ventricular hypertrophy is suggested to be directed inferiorly ${ }^{1 /}$ in contrast with that of inferior myocardial infarction because of clockwise rotation of the QRS loop in the horizontal plane.

The initial 30-msec QRS segmental polar vectors were also more sensitive to detect abnormalities in inferior myocardial infarction than the mean QRS polar vector although the improvement was not as much as in anterior myocardial infarction. This observation denotes that not only the polar vector of the whole QRS loop, but also of the initial part of the QRS loop was directed posteriorly in the majority of inferior myocardial infarction (Figs. 2 and 3 ).

There was no significant difference in the magnitude of the initial 30msec QRS segmental polar vector between inferior myocardial infarction and the normal, while the mean values (mean $\pm 1 \mathrm{SD}\left(\mathrm{mV}^{2}\right)$ ) of the 3 components of the vector were significantly smaller in inferior myocardial infarction $(\mathrm{Vx}=$ $0.048 \pm 0.070, \quad \mathrm{~V} y=0.140 \pm 0.167, \quad \mathrm{~V} z=-0.113 \pm 0.070$ in IMI, vs $\mathrm{Vx}=$ $0.121 \pm 0.102, V y=0.171 \pm 0.113, V z=-0.015 \pm 0.054$ in normal) except for $V_{y}$ which was the area of the initial 30-msec portion of the QRS loop in the horizontal plane. These values explain the posterior deviation of the initial 30-msec segmental polar vector which corresponds with the clockwise inscription of the QRS loop in the frontal plane in inferior myocardial infarction.

In the preliminary study, ${ }^{11}$ ) it was evaluated that which of the initial (e.g. 10-, 20-, 30-, 40-, or 50-msec) segmental polar vectors were useful for the separation between normal and myocardial infarction. The initial 30msec QRS segmental polar vector was confirmed to be the most sensitive discriminator among above mentioned segmental polar vectors because of the relatively narrow range in its direction, while the normal range of the initial 10 - or 20-msec QRS segmental polar vector was too wide in the direction to discriminate myocardial infarction as shown in Table $\mathrm{I}$.

Since Schellong's observation, ${ }^{8 /}$ Milnor also confirmed that the normal spatial loop approached ideal planarity, and gave a criterion characterizing the position of the QRS plane in space. ${ }^{16)}$ Since then, several indicators were analysed for the loop planarity. Pipberger and Carter ${ }^{11}$ obtained "edge- 
wise" and "broadside" spatial loop by means of a lead resolver of the Schmitt-type. ${ }^{17)}$ They mentioned that the ratio between major and minor axes of "edgewise" projection was an indicator of the planarity of the QRS loops and found that 3.8 was the upper normal limit for this ratio; 3.3 was the limit established in this study. Later, Draper et $\mathrm{al}^{\left.{ }^{\prime}\right)}$ obtained Eigenvectors $^{\mathbf{1 8})}$ through a least square fitting procedure and estimated the planarity of the loop by Eigenvector ratios. Rijlant ${ }^{19)}$ defined the planarity as the ratio between the maximal and minimal axes in an edgewise projection. Okamoto and Simonson ${ }^{7}$ calculated the mean planarity of the QRSsE or TsE loop given by the ratio between the mean distance of the instantaneous perpendicular projections to the major QRS or $\mathrm{T}$ plane and the magnitude of the spatial maximal QRS or $T$ vector, and found that the planarity index was of relatively poor diagnostic quality. In Japan, Nomura and colleagues presented the planarity of the spatial loop by the root mean square of an included angle between the approximated plane of the loop and each instantaneous vector. ${ }^{2}$ All these reports including Ohshita's ${ }^{20)}$ confirmed the planarity of QRS or $\mathrm{T}$ loops.

In this study, the ratio of thickness to length was used for a planarity index and width to length ratio for characterizing spatial loop configuration. Although these measurements did not lead to recognition of high percentages of abnormality in myocardial infarction, anterior myocardial infarction was characterized by the finding that the QRS loop was narrow and poor in its planarity compared with the normal. These changes and deformities of the QRS loop may be due to the localized decrease in cardiac electromotive forces or conduction disturbances caused by myocardial infarction. ${ }^{211,22)}$

The normal spatial $\mathrm{T}$ loop is generally elliptical, clongated, and smooth, ${ }^{23}$ and a wide $T$ loop can be regarded as a manifestation of myocardial ischemia, although $\mathrm{T}$ loop changes themselves are not specific to justify the diagnosis of myocardial infarction. ${ }^{24}$ )

Using the length to width ratio $(\mathrm{L} / \mathrm{W})$, Chou et $\mathrm{al}^{25)}$ quantitated the widening of the spatial $T$ loop, although they obtained the length as the largest maximal $T$ vector among the 3 planes and the width as the largest maximal width among the 3 planes. This ratio gives a closer approximation to the true length to width ratio of the spatial loop. They took $\mathrm{L} / \mathrm{W}$ ratio of 2.6 or less (i.e., $\mathrm{W} / \mathrm{L}$ ratio of 0.38 or more) as abnormal, and demonstrated that the incidence of the abnormally wide TsE loop was higher in myocardial infarction and ventricular hypertrophy. In their study, 24 of 138 cases $(17.4 \%$ ) with myocardial infarction had the abnormally wide $\mathrm{T}$ loop.

In the present study, the value, 0.53 was taken as the upper limit of $\mathrm{W} / \mathrm{L}$. ratio and the $T$ loop which exceeded the value was considered to be ab- 
normally wide or "circular". The calculation was based on the measurements from the spatial loop itself instead of those of its plane projections. This study confirmed that the abnormally wide $\mathrm{T}$ loop was seen in 36 of 69 cases $(52.2 \%)$ in anterior myocardial infarction and 4 of 32 cases $(13 \%)$ in inferior myocardial infarction. The results were consistent with the reports by $\mathrm{Mori}^{261}$ and $\mathrm{Ide}^{27)}$ who also demonstrated the higher incidence of the wide $\mathrm{T}$ loop in anterior myocardial infarction $(46 \%)$ than in inferior myocardial infarction (18\%).

Abnormally wide TsE loop is considered to be caused by a change in the sequence of repolarization from the myocardial lesion around the necrotic area. Higher incidence of the wide $\mathrm{T}$ loop and larger mean value of $\mathrm{W} / \mathrm{L}$ ratio in anterior myocardial infarction may be related to the difference in the influence on the instantaneous resultant vector of the repolarization process between anterior (or including septal or lateral) and inferior walls of the left ventricle.

The normal range of the $\mathrm{W} / \mathrm{L}$ ratio calculated in this study was remarkably similar to that reported by Sotobata ${ }^{28}$ who also approximated the value of the $\mathrm{T}$ loop in the 3 plane projections as Chou et al had done. ${ }^{24}$

As pointed out by Okamoto, ${ }^{7)}$ the normal spatial T loop was much better in its planarity than the normal QRS loop. The $\mathrm{T}$ loop planarity was poorer in anterior myocardial infarction than inferior myocardial infarction probably due to the smaller mean values of the length in anterior myocardial infarction.

In conclusion, analysis of the spatial loop in its own reference frame based on the polar vector was useful to characterize the spatial orientation of the loop, the loop shape and sense of inscription of the loop. In addition, the initial segmental QRS polar vector was proved to be a sensitive indicator of mild localized abnormalities of the initial portion of the spatial QRS loop, which led to a higher recognition rate of abnormality in myocardial infarction than the mean QRS polar vector. The information which was obtained automatically by using a mathematical procedure in the present study appears to be directly applicable to clinical diagnosis of the ECG with an aid of a computer.

\section{ACKNOWLEDGMENTS}

The author wishes to express his gratitude to Professor Itsuro Sobue and Professor Kazuo Yamada for their proper advice, and Dr. Shoji Yasui, Dr. Iwao Sotobata, Dr. Yoshihiko Watanabe, and Dr. Noboru Okamoto for their kind guidance and suggestion throughout the study. 


\section{REFERENCES}

1. Pipberger HV, Carter TN: Analysis of the normal and abnormal vectorcardiogram in its own reference frame. Circulation 25: 827, 1962

2. Nomura $\mathrm{Y}$, Takaki $\mathrm{X}$, Toyama S: Computer analysis of the spatial vectorcardiograms with Frank's lead system. Jap Circulat J 31: 1441, 1967

3. Burger HC, Vaane JP: A criterion characterizing the orientation of a vectorcardiogram in space. Am Heart J 56: 29, 1958

4. Yamauchi $\mathrm{K}$ et al: Sex and age differences in polar vectors (Frank lead system) in normal Japanese (abstr). Jap Circulat J 37: 879, 1973

5. Yokoi M, Okamoto N, Mizuno Y, Uozumi Z, Iwatsuka T, Takahashi H, Yasui S, Yamauchi $\mathrm{K}$, Watanabe $\mathrm{Y}$ : Automatic ECG diagnosis in rapid mass screening. Jap J Med Elec \& Biol Engng 11: 195, 1973

6. Simonson E: Differentiation between Normal and Abnormal in Electrocardiography. The CV Mosby Co, St Louis, 1961

7. Okamoto N, Simonson E: Separation of normal and abnormal vectorcardiograms. Use of spatial orientation of initial, central and terminal segmental planes of the QRS loop. Am J Cardiol 18: 682, 1966

8. Schellong F: Grundzüge einer Klinischen Vektordiagraphie des Herzens. Spriger-Verlag, Berlin, 1937

9. Draper HW, Peffer CJ, Stallmann FW, Littman D, Pipberger HV: The corrected orthogonal electrocardiogram and vectorcardiogram in 510 normal men (Frank lead system). Circulation 30: 853,1964

10. Einthoven W, Fahr G, DE Waart A: Über die Richtung und die manifeste Grösse der Potentialschwankungen im menschlichen Herzen und über die Einfluss der Herzlage auf dic Form des Elektrokardiograms. Pflügers Arch ges Physiol 150: 275, 1913

11. Pipberger HV, Stallmann FW, Yano K, Draper HW: Digital computer analysis of the normal and abnormal electrocardiogram. Prog Cardiovasc Dis 5: 378, 1963

12. Pipberger HV, Stallmann FW: Computation of differential diagnosis in electrocardiography. Ann New York Acade Sc 115: 1115, 1964

13. Yasui S, Yamauchi K, Yokota M, Tanimura H, Watanabe Y, Sotobata I, Yokoi M, Okamoto $\mathrm{N}$ : Computer analysis of vectorcardiograms. With special reference to segmental polar vector in myocardial infarction. Electrocardiography Adv Cardiol 16: 424, 1976

14. Chou TC, Helm RA, Kaplan S: Clinical Vectorcardiography. 2nd Ed, Grune \& Stratton, New York, 1974

15. Yamauchi $\mathrm{K}$ et al: A study on vectorcardiograms of myocardial infarction. Analysis of polar vector (abstr). Jap J Geriatrics (Suppl 10): 61, 1973

16. Milnor WR: The normal vectorcardiogram and a system for the classification of vectorcardiographic abnormalities. Circulation 16: 95, 1957

17. Schmitt OH: Cathod-ray presentation of three dimensional data. J Appl Physics 18: 819, 1947

18. Brinberg L: Quantitative Vector Electrocardiography. Waverly Prcss, Baltimore, 1960

19. Rijlant P: L'électrogenèse globale du coeur chez l'homme. Electrocardiographie vectrielle et vectorcardiographie. Acta Cardiol 13: 349, 1958

20. Ohshita $\mathrm{K}$ : Study on vectorcardiogram, pianarity of normal spatial vectorcardiogram. Jap Circulat J 37: 879, 1973

21. Wang J: A quantitative analysis of the spatial characteristics in the QRS loop of the Frank system VCG in myocardial infarction. Fukuoka Acta Med 61: 341, 1970

22. Gunnar RM, Pietras RJ, Blackaller J, Dadmun SE, Szanto PB, Tobin JR: Correlation of vectorcardiographic criteria for myocardial infarction with autopsy findings. Circulation 35 : 158,1967

23. Winsor T: Primer of Vectorcardiography. Lea \& Febiger, Philadelphia, 1972 
24. Eddleman EE, Pipberger HV: Computer analysis of the orthogonal electrocardiogram and vectorcardiogram in 1002 patients with myocardial infarction. Am Heart J 81: 608, 1971

25. Chou TC, Helm RA, Lach R: The significance of a wide TsE loop. Circulation 30: 400, 1964

26. Mori $\mathrm{H}$, Ohshita $\mathrm{K}$ : Diagnosis of myocardial infarction: electrocardiograms, vectorcardiograms and its analog computer analysis. Jap Circulat J 31: 1644, 1967

27. Ide $\mathrm{T}$ : Studies on the $\mathrm{T}$ waves in the spatial velocity electrocardiography of myocardial infarction. Jap Circulat J 33: 1343, 1969

28. Sotobata I: A study on vectorcardiograms of Japanese normal male adults. Analysis of TsE loops. Jap Circulat J 31: 665, 1967 